\title{
AN EXTENSION OF THE CLARK-OCONE FORMULA
}

\author{
SAID NGOBI and AUREL STAN
}

Received 21 April 2003

\begin{abstract}
A white noise proof of the classical Clark-Ocone formula is first provided. This formula is proven for functions in a Sobolev space which is a subset of the space of square-integrable functions over a white noise space. Later, the formula is generalized to a larger class of operators.
\end{abstract}

2000 Mathematics Subject Classification: 60H40, 60H20, 60H05, 60G15.

1. Introduction. Consider a probability space $(\Omega, \mathscr{F}, P)$. Let $B(t)$ be a Brownian motion, $0 \leq t \leq 1$, and $\mathscr{F}_{t}=\sigma\{B(s) \mid 0 \leq s \leq t\}$ the filtration it generates. In [1,7], the following results are considered. If $F(B(t))$ is any finite functional of Brownian motion, then it can be represented as a stochastic integral. This representation is not unique. However, if $E\left(F^{2}(B(\cdot))\right)<\infty$, then according to Martingale representation theory, $F$ does have a unique representation as the sum of a constant and an Itô stochastic integral:

$$
F=E(F)+\int_{0}^{1} \phi(t) d B(t)
$$

where the process $\phi$ belongs to the space $L^{2}([0,1] \times \Omega ; \mathbb{R})$ and, for all $t \in[0,1]$, the random variable $\phi(t)$ is $\mathscr{F}_{t}$-measurable. It is also shown in the same papers that if $F$ is Fréchet-differentiable and satisfies certain technical regularity conditions, then $F$ has an explicit expression as a stochastic integral in which the integrand consists of the conditional expectations of the Fréchet differential. It is this explicit representation for the integrand that gives rise to the well-known Clark-Ocone formula.

In the white noise setup, if we replace Fréchet differentiability with white noise differentiation, then we need the condition that $F \in \mathcal{W}^{1 / 2}$, a Sobolev space which is a subset of the space of square-integrable functions, for the result to have a meaning. In [5], the authors extended the Clark-Ocone formula to generalized Wiener functionals. The formula is the same and only the space on which it holds is enlarged. In this paper, we generalize the formula by using the differential operators $D_{e_{t}}$ and their adjoints $D_{f_{t}}^{*}$ for any families of temperate distributions $\left\{e_{t}\right\}_{t \in \mathbb{R}}$ and $\left\{f_{t}\right\}_{t \in \mathbb{R}}$ satisfying certain technical conditions. In so doing, we regard the formula as the equality of two operators and extend it to a larger class of operators. When $e_{t}=f_{t}=\delta_{t}$ (the Dirac measure at $t$ ), for all $t \in \mathbb{R}$, we obtain the classical Clark-Ocone formula. For purposes of continuity of our argument, we first verify the results in $[1,7]$ using the $S$-transform, which really looks similar to the one found in [5]. 
2. Background and notations. In [4], various constructions of Gel'fand triples are presented. We will be using in this paper the triples $\mathscr{S}(\mathbb{R}) \subset L^{2}(\mathbb{R}) \subset \mathscr{Y}^{\prime}(\mathbb{R})$ and $(\mathscr{Y}(\mathbb{R})) \subset$ $\left(L^{2}\right) \subset(\mathscr{Y}(\mathbb{R}))^{*}$, where $\mathscr{Y}(\mathbb{R})$ is the Schwartz space and $\left(L^{2}\right)=L^{2}\left(\mathscr{Y}^{\prime}(\mathbb{R})\right)$.

For any $\delta_{t} \in \mathscr{Y}^{\prime}(\mathbb{R})$, according to the notations in [4, 6], the white noise differential operator $\partial_{t}$ and its adjoint $\partial_{t}^{*}$ are defined by the duality between $(\mathscr{Y}(\mathbb{R}))$ and $(\mathscr{Y}(\mathbb{R}))^{*}$ as

$$
\left\langle\left\langle\partial_{t}^{*} \Phi, \varphi\right\rangle\right\rangle=\left\langle\left\langle\Phi, \partial_{t} \varphi\right\rangle\right\rangle, \quad \Phi \in(\mathscr{Y}(\mathbb{R}))^{*}, \varphi \in(\mathscr{S}(\mathbb{R})) .
$$

In the same references, for any $x \in \mathscr{S}(\mathbb{R})_{c}$, the exponential function : $e^{\langle\cdot, x\rangle}:$ is defined by the formula

$$
: e^{\langle\cdot, x\rangle}:=\sum_{n=0}^{\infty} \frac{1}{n !}\left\langle:{ }^{\otimes n}:, x^{\otimes n}\right\rangle,
$$

where

$$
: x^{\otimes n}:=\sum_{k=0}^{[n / 2]}\left(\begin{array}{c}
n \\
2 k
\end{array}\right)(2 k-1) ! !(-1)^{k} x^{\otimes(n-2 k)} \hat{\otimes} T^{\otimes k}
$$

with $\tau$ being the trace operator from $\mathscr{Y}(\mathbb{R}) \otimes \mathscr{Y}(\mathbb{R})$ into $\mathbb{C}$.

Moreover, the $S$-Transform of $\Phi$ is the function $S(\Phi): \mathscr{Y}(\mathbb{R})_{c} \rightarrow \mathbb{C}$ defined by

$$
S(\Phi)(\xi)=\left\langle\left\langle\Phi,: e^{\langle\cdot, \xi\rangle}:\right\rangle\right\rangle, \quad \xi \in \mathscr{S}(\mathbb{R})_{c} .
$$

Note also that

$$
S(\Phi \diamond \Psi)=(S \Phi)(S \Psi)
$$

where $\diamond$ denotes the Wick product.

Definition 2.1. Let $\varphi:[a, b] \rightarrow(\varphi(\mathbb{R}))^{*}$ be Pettis-integrable. The white noise integral $\int_{a}^{b} \partial_{t}^{*} \varphi(t) d t$ is called the Hitsuda-Skorokhod integral of $\varphi$ if it is a random variable in $\left(L^{2}\right)$.

The following theorem is due to Kubo and Takenaka [3]. (See also [4].)

THEOREM 2.2. Let $\varphi(t)$ be a stochastic process in the space $L^{2}\left([a, b] \times \mathscr{Y}^{\prime}(\mathbb{R})\right)$ which is nonanticipating and $\int_{a}^{b}\|\varphi(t)\|_{0}^{2} d t<\infty$. Then the function $\partial_{t}^{*} \varphi(t), t \in[a, b]$, is Pettisintegrable and

$$
\int_{a}^{b} \partial_{t}^{*} \varphi(t) d t=\int_{a}^{b} \varphi(t) d B(t)
$$

where the right-hand side is the Itô integral of $\varphi$.

It is noted from the above theorem that the Hitsuda-Skorokhod integral is an extension of the Itô integral. (See [4] for examples of the Hitsuda-Skorokhod integral.) 
DeFinition 2.3. For $\varphi=\sum_{n=0}^{\infty}\left\langle: \cdot{ }^{\otimes n}:, f_{n}\right\rangle$, define

$$
N \varphi=\sum_{n=1}^{\infty} n\left\langle: \cdot{ }^{\otimes n}:, f_{n}\right\rangle .
$$

The operator $N$ is called the number operator. Moreover, the power $N^{r}, r \in \mathbb{R}$, of the number operator is defined in the following way: for $\varphi=\sum_{n=0}^{\infty}\left\langle: \cdot{ }^{\otimes n}:, f_{n}\right\rangle$,

$$
N^{r} \varphi=\sum_{n=1}^{\infty} n^{r}\left\langle:{ }^{\otimes n}:, f_{n}\right\rangle .
$$

For any $r \in \mathbb{R}, N^{r}$ is a continuous linear operator from $(\mathscr{Y}(\mathbb{R}))$ into itself and from $(\mathscr{Y}(\mathbb{R}))^{*}$ into itself. Let $\mathcal{W}^{1 / 2}$ be the Sobolev space of order $1 / 2$ for the Gel'fand triple $(\mathscr{S}(\mathbb{R})) \subset\left(L^{2}\right) \subset(\mathscr{S}(\mathbb{R}))^{*}$. In other words, $\mathscr{W}^{1 / 2}$ will denote the set of $\varphi \in\left(L^{2}\right)$ such that $\left(\partial_{t} \varphi\right)_{t \in \mathbb{R}} \in L^{2}\left(\mathbb{R} ;\left(L^{2}\right)\right)$. Thus

$$
\mathcal{W}^{1 / 2}=\left\{\varphi \in\left(L^{2}\right) \mid \int_{\mathbb{R}}\left\|\partial_{t} \varphi\right\|_{0}^{2} d t<\infty\right\} .
$$

The norm on $W^{1 / 2}$ will be defined as

$$
\|\varphi\|_{1 / 2}^{2}:=\|\varphi\|_{0}^{2}+\int_{\mathbb{R}}\left\|\partial_{t} \varphi\right\|_{0}^{2} d t .
$$

ObSERVATION 2.4. If $\varphi \in\left(L^{2}\right)$, then

$$
\int_{\mathbb{R}}\left\|\partial_{t} \varphi\right\|_{0}^{2} d t=\left\|N^{1 / 2} \varphi\right\|_{0}^{2} .
$$

Proof. The proof can be found in [4].

LEMMA 2.5. $(\mathscr{Y}(\mathbb{R})) \subset W^{1 / 2}$.

Proof. See $[4,6]$.

3. An extension of the Clark-Ocone formula. In what follows, we deal with an arbitrary Gel'fand triple $\mathscr{E} \subset E \subset \mathscr{E}^{\prime}$.

Notation 3.1. Let $K$ be a closed subspace of $E$ and $K_{c}$ the complexification of $K$. Let $\left(L_{K}^{2}\right)$ be the subspace of $\left(L^{2}\right)$ consisting of all functions $\varphi=\sum_{n=0}^{\infty}\left\langle:{ }^{\otimes n}:, f_{n}\right\rangle$ such that $f_{n} \in K_{c}^{\hat{\otimes} n}$ for all $n \geq 0$.

For $p \geq 0$, define

$$
\left(\mathscr{E}_{K}\right)_{p}=\left\{\varphi \in\left(L_{K}^{2}\right) \mid\|\varphi\|_{p}<\infty\right\}
$$

Let $\left(\mathscr{E}_{K}\right)_{-p}$ be the completion of $\left(L_{K}^{2}\right)$ with respect to the norm $\|\cdot\|_{-p}$. Thus, for all $p \in \mathbb{R}$, we have $\left(\mathscr{E}_{K}\right)_{p} \subseteq(\mathscr{E})_{p}$. Let

$$
\left(\mathscr{E}_{K}\right)=\bigcap_{p \geq 0}\left(\mathscr{E}_{K}\right)_{p} \subseteq(\mathscr{E}), \quad\left(\mathscr{E}_{K}\right)^{*}=\bigcup_{p \geq 0}\left(\mathscr{E}_{K}\right)_{-p} \subseteq(\mathscr{E})^{*}
$$


Definition 3.2. A function $\phi \in(\mathscr{E}) *$ is said to be supported by $K$ if $\phi \in\left(\mathscr{E}_{K}\right)^{*}$.

LEMMA 3.3. Let $H_{1}$ and $H_{2}$ be two orthogonal closed subspaces of E. Suppose $\phi$ and $\psi \in\left(L^{2}\right)$ are supported by $H_{1}$ and $H_{2}$, respectively. Then $\phi \diamond \psi=\phi \cdot \psi$ and

$$
\|\phi \diamond \psi\|_{0}=\|\phi\|_{0} \cdot\|\psi\|_{0}
$$

Proof. This can be done via the $\mathscr{S}$-transform. See [4].

DEFINITION 3.4. Let $B: E \rightarrow E$ be a bounded linear operator. The second quantization operator of $B, \Gamma(B):\left(L^{2}\right) \rightarrow(\mathscr{E}) *$ for $\varphi=\sum_{n=0}^{\infty}\left\langle:{ }^{\otimes n}:, f_{n}\right\rangle$, is defined as

$$
\Gamma(B) \varphi=\sum_{n=0}^{\infty}\left\langle: \cdot{ }^{\otimes n}:, B^{\otimes n} f_{n}\right\rangle .
$$

It is easy to see that

$$
\|\Gamma(B) \varphi\|_{0}^{2} \leq \sum_{n=0}^{\infty} n !\|B\|^{2 n}\left|f_{n}\right|_{0}^{2} .
$$

Therefore, if $\|B\| \leq 1$, then $\Gamma(B)$ is a bounded linear operator from $\left(L^{2}\right)$ into $\left(L^{2}\right)$ of norm less than or equal to 1 .

On the other hand, if $\|B\|>1$, then $\Gamma(B)$ is a bounded linear operator from $\left(L^{2}\right)$ into $\left(\mathscr{E}_{-}\right)$), choosing $p>0$ sufficiently large such that $\lambda_{1}^{p} \geq\|B\|$.

OBSERVATION 3.5. If $B: E \rightarrow E$ is a bounded linear operator and $\xi \in E_{c}$, then

$$
\Gamma(B): e^{\langle\cdot, \xi\rangle}:=: e^{\langle\cdot, B \xi\rangle}: .
$$

\section{ProOF.}

$$
\begin{aligned}
\Gamma(B): e^{\langle\cdot, \xi\rangle}: & =\Gamma(B)\left(\sum_{n=0}^{\infty} \frac{1}{n !}\left\langle:{ }^{\otimes n}:, \xi^{\otimes n}\right\rangle\right) \\
& =\sum_{n=0}^{\infty} \frac{1}{n !}\left\langle:{ }^{\otimes n}:, B^{\otimes n} \xi^{\otimes n}\right\rangle \\
& =\sum_{n=0}^{\infty} \frac{1}{n !}\left\langle:{ }^{\otimes n}:,(B \xi)^{\otimes n}\right\rangle=: e^{\langle\cdot, B \xi\rangle}: .
\end{aligned}
$$

We now switch back to the Gel'fand triple $\mathscr{S}(\mathbb{R}) \subset L^{2}(\mathbb{R}) \subset \mathscr{Y}^{\prime}(\mathbb{R})$.

If $g \in L^{\infty}(\mathbb{R})$, then $g$ can be identified with the multiplication operator $T_{g}: L^{2}(\mathbb{R}) \rightarrow$ $L^{2}(\mathbb{R})$ defined by $T_{g} f=g f$. This is a bounded operator of norm equal to $\|g\|_{\infty}$. We will denote $\Gamma\left(T_{g}\right)$ simply by $\Gamma(g)$.

Let $\mathscr{B}\left(S^{\prime}(\mathbb{R})\right)$ be the $\sigma$-field generated by the open subsets of $S^{\prime}(\mathbb{R})$. If $\left\{f_{i}\right\}_{i \in I}$ is a family of complex-valued $\mathscr{B}\left(S^{\prime}(\mathbb{R})\right)$-measurable functions defined on $S^{\prime}(\mathbb{R})$, then we denote by $\sigma\left\{f_{i} \mid i \in I\right\}$ the smallest $\sigma$-field $\mathscr{F}_{F}$ contained in $\mathscr{B}\left(S^{\prime}(\mathbb{R})\right)$ such that, for all $i \in I, f_{i}$ is $\mathscr{F}$-measurable.

If $\mathscr{F}$ is a sub- $\sigma$-field of $\mathscr{B}\left(S^{\prime}(\mathbb{R})\right)$ and $\varphi \in\left(L^{2}\right)$, then we denote by $E(\varphi \mid \mathscr{F})$ the conditional expectation of $\varphi$ with respect to $\mathscr{F}$. 
Let

$$
B(t)= \begin{cases}\left\langle\cdot, 1_{[0, t]}\right\rangle & \text { if } t \geq 0, \\ -\left\langle\cdot, 1_{[t, 0]}\right\rangle & \text { if } t<0 .\end{cases}
$$

It is shown in [4] that $\{B(t)\}_{t \in \mathbb{R}}$ is a Brownian-motion process.

LEMMA 3.6. Let $\varphi \in\left(L^{2}\right)$ and $t \in \mathbb{R}$. Let $\mathscr{F}_{t}=\sigma\{B(s) \mid s \leq t\}$. Then

$$
E\left(\varphi \mid \mathscr{F}_{t}\right)=\Gamma\left(1_{(-\infty, t]}\right) \varphi .
$$

Proof. For the proof plus other related properties of the second quantization operator, see $[2,8]$.

The following theorem provides a white noise proof of the Clark-Ocone formula for functions in the Sobolev space $W^{1 / 2}$.

THEOREM 3.7 (the Clark-Ocone formula). Let $W^{1 / 2}$ be the Sobolev space from the Gel'fand triple $(\mathscr{Y}(\mathbb{R})) \subset\left(L^{2}\right) \subset(\mathscr{Y}(\mathbb{R}))^{*}$. Suppose $B(t)$ is the Brownian motion given by $B(t)=\left\langle\cdot, 1_{[0, t]}\right\rangle, t \in \mathbb{R}$, with $\mathscr{F}_{t}=\sigma\{B(s) \mid s \leq t\}$ the filtration it generates. Then, for any $\phi \in \mathcal{W}^{1 / 2}$, the following formula holds:

$$
\phi=E(\phi)+\int_{\mathbb{R}} E\left(\partial_{t} \phi \mid \mathscr{F}_{t}\right) d B(t) .
$$

Proof. We can rewrite the stochastic integral in the above equation as

$$
\int_{\mathbb{R}} E\left(\partial_{t} \phi \mid \mathscr{F}_{t}\right) d B(t)=\int_{\mathbb{R}} \partial_{t}^{*} E\left(\partial_{t} \phi \mid \mathscr{F}_{t}\right) d t .
$$

Since $E\left(\partial_{t} \phi \mid \mathscr{F}_{t}\right)=\Gamma\left(1_{(-\infty, t]}\right) \partial_{t} \phi,(3.10)$ is equivalent to

$$
\phi=E(\phi)+\int_{\mathbb{R}} \partial_{t}^{*} \Gamma\left(1_{(-\infty, t]}\right) \partial_{t} \phi d t
$$

where the integral in (3.12) is regarded as a white noise integral in the Pettis sense. We will prove formula (3.12) using the $S$-transform.

Let $\phi \in W^{1 / 2}$ be fixed. Because $\int_{\mathbb{R}}\left\|\partial_{t} \phi\right\|_{0}^{2} d t<\infty$, we conclude that there exists a subset $N$ of $\mathbb{R}$, of Lebesgue measure zero, such that for all $t \in \mathbb{R} \backslash N,\left\|\partial_{t} \phi\right\|_{0}<\infty$. This means that for all $t \in \mathbb{R} \backslash N, \partial_{t} \phi \in\left(L^{2}\right)$. By the boundedness of the operator $\Gamma\left(1_{(-\infty, t]}\right)$, we conclude that for all $t \in \mathbb{R} \backslash N, \Gamma\left(1_{(-\infty, t]}\right) \partial_{t} \phi \in\left(L^{2}\right)$ and $\partial_{t}^{*} \Gamma\left(1_{(-\infty, t]}\right) \partial_{t} \phi \in(\mathscr{S}(\mathbb{R}))^{*}$. We define the function $f: \mathbb{R} \rightarrow(\mathscr{Y}(\mathbb{R}))^{*}$ by

$$
f(t)= \begin{cases}\partial_{t}^{*} \Gamma\left(1_{(-\infty, t]}\right) \partial_{t} \phi & \text { if } t \in \mathbb{R} \backslash N, \\ 0 & \text { if } t \in N .\end{cases}
$$

By abuse of notation, we will write $f(t)=\partial_{t}^{*} \Gamma\left(1_{(-\infty, t]}\right) \partial_{t} \phi$ for all $t \in \mathbb{R}$. First, we will check that the function $f$ is Pettis-integrable. To see this, we observe first that for any $\varphi \in(\mathscr{Y}(\mathbb{R}))$, the function $t \mapsto\left\langle\left\langle\partial_{t}^{*} \Gamma\left(1_{(-\infty, t]}\right) \partial_{t} \phi, \varphi\right\rangle\right\rangle$, which is the same as the function $t \mapsto\left\langle\left\langle\Gamma\left(1_{(-\infty, t]}\right) \partial_{t} \phi, \partial_{t} \varphi\right\rangle\right\rangle$, is measurable. Thus, $f$ is weakly measurable. It is also easy to see that $\langle\langle f(\cdot), \varphi\rangle\rangle \in L^{1}(\mathbb{R})$ for all $\varphi \in(\mathscr{Y}(\mathbb{R}))$. Hence, $f$ is Pettis-integrable. 
We start proving (3.10) first for exponential functions $\phi=F_{\eta}=\sum_{n=0}^{\infty}(1 / n !)\left\langle: \cdot{ }^{\otimes n}\right.$ :, $\left.\eta^{\otimes n}\right\rangle, \eta \in L^{2}(\mathbb{R})_{c}$. First, we note that $F_{\eta} \in W^{1 / 2}$ because

$$
\begin{aligned}
\sum_{n=1}^{\infty} n n !\left|\frac{\eta^{\otimes n}}{n !}\right|_{0}^{2} & =\sum_{n=1}^{\infty} \frac{n}{n !}|\eta|_{0}^{2 n} \\
& =|\eta|_{0}^{2} \sum_{n=1}^{\infty} \frac{1}{(n-1) !}|\eta|_{0}^{2(n-1)} \\
& =|\eta|_{0}^{2} e^{|\eta|_{0}^{2}}<\infty .
\end{aligned}
$$

Second, to show that $F_{\eta}=E\left(F_{\eta}\right)+\int_{\mathbb{R}} \partial_{t}^{*} \Gamma\left(1_{(-\infty, t]}\right) \partial_{t} F_{\eta} d t$, we have to check that the $S$-transforms of the two sides are equal:

$$
\begin{aligned}
S\left(E\left(F_{\eta}\right)+\int_{\mathbb{R}} \partial_{t}^{*} \Gamma\left(1_{(-\infty, t]}\right) \partial_{t} F_{\eta} d t\right)(\xi) & =S\left(E\left(F_{\eta}\right)\right)(\xi)+S\left(\int_{\mathbb{R}} \partial_{t}^{*} \Gamma\left(1_{(-\infty, t]}\right) \partial_{t} F_{\eta} d t\right)(\xi) \\
& =S(1)(\xi)+\int_{\mathbb{R}} \xi(t) S\left(\Gamma\left(1_{(-\infty, t]}\right) \partial_{t} F_{\eta}\right)(\xi) d t \\
& =1+\int_{\mathbb{R}} \xi(t) S\left(\Gamma\left(1_{(-\infty, t]}\right)\left(\eta(t) F_{\eta}\right)\right)(\xi) d t \\
& =1+\int_{\mathbb{R}} \xi(t) \eta(t) S\left(\Gamma\left(1_{(-\infty, t]}\right) F_{\eta}\right)(\xi) d t .
\end{aligned}
$$

Using the fact that $\Gamma\left(1_{(-\infty, t]}\right) F_{\eta}=F_{1_{(-\infty, t]} \eta}$, we obtain

$$
\begin{aligned}
S\left(E\left(F_{\eta}\right)+\int_{\mathbb{R}} \partial_{t}^{*} \Gamma\left(1_{(-\infty, t]}\right) \partial_{t} F_{\eta} d t\right)(\xi) & =1+\int_{\mathbb{R}} \xi(t) \eta(t) S\left(F_{1_{(-\infty, t]} \eta}\right)(\xi) d t \\
& =1+\int_{\mathbb{R}} \xi(t) \eta(t)\left\langle\left\langle F_{1_{(-\infty, t]} \eta}, F_{\xi}\right\rangle\right\rangle d t
\end{aligned}
$$

Now, using the fact that $\left\langle\left\langle F_{x}, F_{y}\right\rangle\right\rangle=e^{\langle x, y\rangle}$, for all $x, y \in L^{2}(\mathbb{R})$, we get

$$
\begin{aligned}
S\left(E\left(F_{\eta}\right)+\int_{\mathbb{R}} \partial_{t}^{*} \Gamma\left(1_{(-\infty, t]}\right) \partial_{t} F_{\eta} d t\right)(\xi) & =1+\int_{\mathbb{R}} \xi(t) \eta(t) e^{\left\langle 1_{(-\infty, t]} \eta, \xi\right\rangle} d t \\
& =1+\int_{\mathbb{R}} \xi(t) \eta(t) e^{\int_{-\infty}^{t} \eta(s) \xi(s) d s} d t \\
& =1+\int_{-\infty}^{\infty} \frac{d}{d t}\left(e^{\int_{-\infty}^{t} \eta(s) \xi(s) d s}\right) d t \\
& =1+\left.e^{\int_{-\infty}^{t} \eta(s) \xi(s) d s}\right|_{-\infty} ^{\infty} \\
& =1+e^{\int_{-\infty}^{\infty} \eta(s) \xi(s) d s}-1 \\
& =e^{\int_{-\infty}^{\infty} \eta(s) \xi(s) d s} \\
& =e^{\langle\eta, \xi\rangle} \\
& =\left\langle\left\langle F_{\eta}, F_{\xi}\right\rangle\right\rangle \\
& =S\left(F_{\eta}\right)(\xi)
\end{aligned}
$$

Thus, $F_{\eta}=E\left(F_{\eta}\right)+\int_{\mathbb{R}} \partial_{t}^{*} E\left(\partial_{t} F_{\eta} \mid \mathscr{F}_{t}\right) d t$. 
Now, the vector space spanned by the set $\left\{F_{\eta} \mid \eta \in L^{2}(\mathbb{R})_{c}\right\}$ is dense in $\mathscr{W}^{1 / 2}$. Therefore, for any $\phi \in \mathscr{W}^{1 / 2}$, there exists a sequence $\left\{\varphi_{n}\right\}_{n \geq 1}$ in the span of $\left\{F_{\eta} \mid \eta \in L^{2}(\mathbb{R})_{c}\right\}$ such that $\varphi_{n} \rightarrow \phi$ in $W^{1 / 2}$. The norm of $W^{1 / 2}$ is stronger than the norm of $\left(L^{2}\right)$. Thus, $\varphi_{n} \rightarrow \phi$ in $\left(L^{2}\right)$ and $E\left(\varphi_{n}\right) \rightarrow E(\phi)$. We will show that $\int_{\mathbb{R}} \partial_{t}^{*} E\left(\partial_{t} \varphi_{n} \mid \mathscr{F}_{t}\right) d t \rightarrow$ $\int_{\mathbb{R}} \partial_{t}^{*} E\left(\partial_{t} \phi \mid \mathscr{F}_{t}\right) d t$, weakly. Let $\psi \in(\mathscr{P}(\mathbb{R}))$. Then we have

$$
\begin{aligned}
& \left|\left\langle\left\langle\int_{\mathbb{R}} \partial_{t}^{*} E\left(\partial_{t}\left(\varphi_{n}-\phi\right) \mid \mathscr{F}_{t}\right) d t, \psi\right\rangle\right\rangle\right|=\left|\int_{\mathbb{R}}\left\langle\left\langle\partial_{t}^{*} E\left(\partial_{t}\left(\varphi_{n}-\phi\right) \mid \mathscr{F}_{t}\right), \psi\right\rangle\right\rangle d t\right| \\
& \leq \int_{\mathbb{R}}\left|\left\langle\left\langle E\left(\partial_{t}\left(\varphi_{n}-\phi\right) \mid \mathscr{F}_{t}\right), \partial_{t} \psi\right\rangle\right\rangle\right| d t \\
& \leq \int_{\mathbb{R}}\left\|E\left(\partial_{t}\left(\varphi_{n}-\phi\right) \mid \mathscr{F}_{t}\right)\right\|_{0}\left\|\partial_{t} \psi\right\|_{0} d t \\
& \leq \int_{\mathbb{R}}\left\|\partial_{t}\left(\varphi_{n}-\phi\right)\right\|_{0}\left\|\partial_{t} \psi\right\|_{0} d t \\
& \leq \sqrt{\int_{\mathbb{R}}\left\|\partial_{t}\left(\varphi_{n}-\phi\right)\right\|_{0}^{2} d t \int_{\mathbb{R}}\left\|\partial_{t} \psi\right\|_{0}^{2} d t} \\
& \longrightarrow 0 \text {. }
\end{aligned}
$$

Since, for all $n \in \mathbb{N}$, we have

$$
\varphi_{n}=E\left(\varphi_{n}\right)+\int_{\mathbb{R}} \partial_{t}^{*} \Gamma\left(1_{(-\infty, t]}\right) \partial_{t} \varphi_{n} d t
$$

passing to the limit weakly, as $n \rightarrow \infty$, we get

$$
\phi=E(\phi)+\int_{\mathbb{R}} \partial_{t}^{*} \Gamma\left(1_{(-\infty, t]}\right) \partial_{t} \phi d t
$$

In what follows, we generalize the space $W^{1 / 2}$ to the space $W_{q}^{1 / 2}$ by introducing the weight $q^{2 n}$ in calculating the norm of this new space. In [5], the Clark-Ocone formula was extended to generalized Wiener functionals.

Definition 3.8. Let $q>0$. Define

$$
\mathscr{W}_{q}^{1 / 2}=\left\{\varphi \in(\mathscr{P}(\mathbb{R}))^{*} \mid \text { if } \varphi=\sum_{n=0}^{\infty}\left\langle:{ }^{\otimes n}:, f_{n}\right\rangle \text {, then } \sum_{n=1}^{\infty} n n ! q^{2 n}\left|f_{n}\right|_{0}^{2}<\infty\right\} .
$$

If $\varphi \in W_{q}^{1 / 2}$, then we define

$$
\|\varphi\|_{q, 1 / 2}=\sqrt{\sum_{n=1}^{\infty} n n ! q^{2 n}\left|f_{n}\right|_{0}^{2}}
$$

THEOREM 3.9. Let $v$ be a measure on the Borel subsets of $\mathbb{R}$. If $v$ is absolutely continuous with respect to the Lebesgue measure $d t$ and its Radon-Nikodym derivative $g=d v / d t \in L^{\infty}(\mathbb{R})$, then, for all functions $\varphi \in \mathcal{W}_{\|\mathcal{g}\|_{\infty}}^{1 / 2}$,

$$
\Gamma(g) \varphi=\Gamma(0) \varphi+\int_{\mathbb{R}} \partial_{t}^{*} \Gamma\left(1_{(-\infty, t]} g\right) \partial_{t} \varphi d v(t)
$$


Proof. If $g(x)=0$ almost everywhere, then $v \equiv 0$ and the theorem is trivially true. We assume that $\|\mathcal{g}\|_{\infty}>0$. For all $\psi \in(\mathscr{P}(\mathbb{R}))$, the function $t \mapsto\left\langle\left\langle\partial_{t}^{*} \Gamma\left(1_{(-\infty, t]} \mathcal{g}\right) \partial_{t} \varphi\right.\right.$, $\psi\rangle\rangle$ is measurable. Therefore, the function $t \mapsto \partial_{t}^{*} \Gamma\left(1_{(-\infty, t]} g\right) \partial_{t} \varphi$ is weakly measurable. Also, for all $\psi \in(\mathscr{S}(\mathbb{R}))$, we have

$$
\begin{aligned}
\int_{\mathbb{R}} \mid & \left\langle\left\langle\partial_{t}^{*} \Gamma\left(1_{(-\infty, t]} g\right) \partial_{t} \varphi, \psi\right\rangle\right\rangle \mid d v(t) \\
& =\int_{\mathbb{R}}\left|\left\langle\left\langle\Gamma\left(1_{(-\infty, t]} g\right) \partial_{t} \varphi, \partial_{t} \psi\right\rangle\right\rangle\right| d v(t) \\
& \leq \int_{\mathbb{R}}\left\|\Gamma\left(1_{(-\infty, t]} g\right) \partial_{t} \varphi\right\|_{0}\left\|\partial_{t} \psi\right\|_{0} d v(t) \\
& \leq\left[\int_{\mathbb{R}}\left\|\Gamma\left(1_{(-\infty, t]} g\right) \partial_{t} \varphi\right\|_{0}^{2} d v(t)\right]^{1 / 2}\left[\int_{\mathbb{R}}\left\|\partial_{t} \psi\right\|_{0}^{2} d v(t)\right]^{1 / 2} .
\end{aligned}
$$

Let $\varphi=\sum_{n=0}^{\infty}\left\langle: \cdot{ }^{\otimes n}:, f_{n}\right\rangle$. Then we have

$$
\begin{aligned}
\int_{\mathbb{R}}\left\|\Gamma\left(1_{(-\infty, t]} g\right) \partial_{t} \varphi\right\|_{0}^{2} d v(t) & =\int_{\mathbb{R}} \sum_{n=1}^{\infty}(n-1) !\left|n\left(1_{(-\infty, t]} g\right)^{\otimes(n-1)} f_{n}(t, \cdot)\right|_{0}^{2} d v(t) \\
& =\int_{\mathbb{R}} \sum_{n=1}^{\infty} n^{2}(n-1) !\left|\left(1_{(-\infty, t]} g\right)^{\otimes(n-1)} f_{n}(t, \cdot)\right|_{0}^{2} d v(t) \\
& \leq \int_{\mathbb{R}} \sum_{n=1}^{\infty} n n !|| 1_{(-\infty, t]} g \|_{\infty}^{2(n-1)}\left|f_{n}(t, \cdot)\right|_{0}^{2} d v(t) .
\end{aligned}
$$

Since $d v(t)=g(t) d t$, we obtain

$$
\begin{aligned}
\int_{\mathbb{R}}\left\|\Gamma\left(1_{(-\infty, t]} g\right) \partial_{t} \varphi\right\|_{0}^{2} d v(t) & \leq \int_{\mathbb{R}} \sum_{n=1}^{\infty} n n !\left\|1_{(-\infty, t]} g\right\|_{\infty}^{2(n-1)}\left|f_{n}(t, \cdot)\right|_{0}^{2}\|g\|_{\infty} d t \\
& \leq \int_{\mathbb{R}} \sum_{n=1}^{\infty} n n !\|g\|_{\infty}^{2(n-1)}\left|f_{n}(t, \cdot)\right|_{0}^{2}\|g\|_{\infty} d t \\
& =\sum_{n=1}^{\infty} n n !\|g\|_{\infty}^{2 n-1} \int_{\mathbb{R}}\left|f_{n}(t, \cdot)\right|_{0}^{2} d t \\
& =\sum_{n=1}^{\infty}\|g\|_{\infty}^{2 n-1} n n !\left|f_{n}\right|_{0}^{2} \\
& =\left.\frac{1}{\|g\|_{\infty}}\|\varphi\|\right|_{\|\mathfrak{g}\|_{\infty}, 1 / 2} ^{2} \\
& <\infty .
\end{aligned}
$$

Similarly, we have

$$
\int_{\mathbb{R}}\left\|\partial_{t} \psi\right\|_{0}^{2} d v(t) \leq \int_{\mathbb{R}}\left\|\partial_{t} \psi\right\|_{0}^{2}\|g\|_{\infty} d t \leq\|g\|_{\infty} \int_{\mathbb{R}}\left\|\partial_{t} \psi\right\|_{0}^{2} d t<\infty
$$

Thus, $\int_{\mathbb{R}} \partial_{t}^{*} \Gamma\left(1_{(-\infty, t]} g\right) \partial_{t} \varphi d v(t)$ exists in the Pettis sense. 
For any $\theta \in \mathscr{S}^{\prime}(\mathbb{R})_{c}$ we denote $F_{\theta}=: e^{\langle\cdot, \theta\rangle}:$. We will prove first the theorem for an exponential function $F_{\eta}$, where $\eta \in L^{2}(\mathbb{R})_{c}$. To prove the above equality, we use the $S$-transform. Let $\xi \in \mathscr{S}^{\prime}(\mathbb{R})$. We have

$$
\begin{aligned}
S\left(\Gamma(0) F_{\eta}+\int_{\mathbb{R}} \partial_{t}^{*} \Gamma\left(1_{(-\infty, t]} g\right) \partial_{t} F_{\eta} d v(t)\right)(\xi) \\
\quad=S\left(\Gamma(0) F_{\eta}\right)(\xi)+S\left(\int_{\mathbb{R}} \partial_{t}^{*} \Gamma\left(1_{(-\infty, t]} g\right) \partial_{t} F_{\eta} d v(t)\right)(\xi) \\
=1+\int_{\mathbb{R}} S\left(\partial_{t}^{*} \Gamma\left(1_{(-\infty, t]} g\right) \partial_{t} F_{\eta}\right)(\xi) d v(t) \\
=1+\int_{\mathbb{R}}\left\langle\delta_{t}, \xi\right\rangle S\left(\Gamma\left(1_{(-\infty, t]} g\right) \partial_{t} F_{\eta}\right)(\xi) d v(t) \\
=1+\int_{\mathbb{R}} \xi(t) S\left(\Gamma\left(1_{(-\infty, t]} g\right)\left\langle\delta_{t}, \eta\right\rangle F_{\eta}\right)(\xi) d v(t) \\
=1+\int_{\mathbb{R}} \eta(t) \xi(t) S\left(\Gamma\left(1_{(-\infty, t]} g\right) F_{\eta}\right)(\xi) d v(t) .
\end{aligned}
$$

But $\Gamma\left(1_{(-\infty, t]} g\right) F_{\eta}=F_{1_{(-\infty, t]} g \eta}$. Hence, we get

$$
\begin{aligned}
S\left(\Gamma(0) F_{\eta}+\int_{\mathbb{R}} \partial_{t}^{*} \Gamma\left(1_{(-\infty, t]} g\right) \partial_{t} F_{\eta} d v(t)\right)(\xi) \\
=1+\int_{\mathbb{R}} \eta(t) \xi(t) S\left(F_{1_{(-\infty, t]} g \eta}\right)(\xi) d v(t) \\
=1+\int_{\mathbb{R}} \eta(t) \xi(t)\left\langle\left\langle F_{1_{(-\infty, t]} g \eta}, F_{\xi}\right\rangle\right\rangle d v(t) \\
=1+\int_{\mathbb{R}} \eta(t) \xi(t) e^{\left\langle 1_{(-\infty, t]} g \eta, \xi\right\rangle} d v(t) \\
=1+\int_{\mathbb{R}} \eta(t) \xi(t) e^{\left\langle 1_{(-\infty, t]} g \eta, \xi\right\rangle} g(t) d t \\
=1+\int_{\mathbb{R}} g(t) \eta(t) \xi(t) e^{\int_{-\infty}^{t} g(s) \eta(s) \xi(s) d s} d t \\
=1+\left.e^{\int_{-\infty}^{t} g(s) \eta(s) \xi(s) d s}\right|_{-\infty} ^{+\infty} \\
=1+e^{\int_{-\infty}^{+\infty} g(s) \eta(s) \xi(s) d s}-1 \\
=e^{\langle g \eta, \xi\rangle} \\
=S\left(F_{g \eta}\right)(\xi) \\
=S\left(\Gamma(g) F_{\eta}\right)(\xi) .
\end{aligned}
$$

The theorem can then be checked to hold for all $\mathcal{W}_{\|g\|_{\infty}}^{1 / 2}$-functions by a limiting process in the same way as classical Clark-Ocone formula. 
OBSERVATION 3.10. If we think of $1_{(-\infty, t]}$ as being a multiplication operator, then the following equality holds:

$$
1_{(-\infty, t]}=\int_{-\infty}^{t}\left\langle\delta_{s}, \cdot\right\rangle \delta_{s} d s
$$

in the following sense:

$$
\forall f, g \in \mathscr{Y}(\mathbb{R}), \quad \int_{\mathbb{R}} 1_{(-\infty, t]}(s) f(s) g(s) d s=\int_{-\infty}^{t}\left\langle\delta_{s}, f\right\rangle\left\langle\delta_{s}, g\right\rangle d s .
$$

If we regard $1_{(-\infty, t]}$ and $\int_{-\infty}^{t}\left\langle\delta_{s}, \cdot\right\rangle \delta_{s} d s$ as elements of the space $B(\mathscr{Y}(\mathbb{R}) \times \mathscr{Y}(\mathbb{R}), \mathbb{R})$, then the above observation says that these two operators are the same. We may also write $\int_{-\infty}^{t}\left\langle\delta_{s}, \cdot\right\rangle \delta_{s} d s$ in the form $\int_{-\infty}^{t} \delta_{s}^{*} \delta_{s} d s$.

Let $\left\{e_{t}\right\}_{t \in \mathbb{R}} \subset \mathscr{Y}^{\prime}(\mathbb{R})$ and $\left\{f_{t}\right\}_{t \in \mathbb{R}} \subset \mathscr{Y}^{\prime}(\mathbb{R})$ such that, for all $h \in \mathscr{Y}(\mathbb{R})$, the functions $t \mapsto\left\langle e_{t}, h\right\rangle$ and $t \mapsto\left\langle f_{t}, h\right\rangle$ are measurable and there exist two positive numbers $u$ and $M$ such that, for all $h \in \mathscr{S}(\mathbb{R})$, we have $\int_{\mathbb{R}}\left|\left\langle e_{t}, h\right\rangle\right|^{2} d t \leq M \cdot|h|_{u}^{2}$ and $\int_{\mathbb{R}}\left|\left\langle f_{t}, h\right\rangle\right|^{2} d t \leq$ $M \cdot|h|_{u}^{2}$. Then we may define the operator $T \in B(\mathscr{Y}(\mathbb{R}) \times \mathscr{Y}(\mathbb{R}), \mathbb{R})$ by the formula

$$
T(g, h)=\int_{\mathbb{R}}\left\langle e_{t}, g\right\rangle \cdot\left\langle f_{t}, h\right\rangle d t .
$$

$T$ is a continuous operator and, since $\mathscr{S}(\mathbb{R})$ is a nuclear space, by the abstract kernel theorem, there exists a unique bounded linear operator $P: \mathscr{Y}(\mathbb{R}) \rightarrow \mathscr{Y}^{\prime}(\mathbb{R})$ such that for all $g, h \in \mathscr{Y}(\mathbb{R})$, we have

$$
T(g, h)=\langle P g, h\rangle
$$

We will write

$$
P=\int_{\mathbb{R}}\left\langle e_{t}, \cdot\right\rangle f_{t} d t
$$

or

$$
P=\int_{\mathbb{R}} f_{t}^{*} e_{t} d t
$$

The abstract kernel theorem further guarantees the existence of two positive numbers $p$ and $q$ such that $P: \mathscr{Y}(\mathbb{R})_{p} \rightarrow \mathscr{Y}^{\prime}(\mathbb{R})_{q}$ is a Hilbert-Schmidt operator, therefore bounded. Thus, there exist $p, q$, and $C>0$ such that

$$
\forall g \in \mathscr{Y}(\mathbb{R}), \quad|P g|_{-q} \leq C \cdot|g|_{p}
$$

We may define the second quantization operator of $P$ as $\Gamma(P):(\mathscr{Y}(\mathbb{R})) \rightarrow(\mathscr{Y}(\mathbb{R}))^{*}$ in the following way: if $\varphi=\sum_{n=0}^{\infty}\left\langle: \cdot{ }^{\otimes n}: g_{n}\right\rangle \in(\mathscr{S}(\mathbb{R}))$, where for all $n \geq 0, g_{n} \in \mathscr{S}(\mathbb{R})_{c}^{\hat{\otimes} n}$, then

$$
\Gamma(P) \varphi=\sum_{n=0}^{\infty}\left\langle: \cdot{ }^{\otimes n}:, P^{\otimes n} g_{n}\right\rangle .
$$


As an observation, $\Gamma(P)$ is a bounded linear operator from $(\mathscr{Y}(\mathbb{R}))$ into $(\mathscr{Y}(\mathbb{R}))^{*}$. For any $t \in \mathbb{R}$, we can also define the operator $P_{t}=\int_{-\infty}^{t}\left\langle e_{s}, \cdot\right\rangle f_{s} d s$ as $P_{t}=\int_{\mathbb{R}}\left\langle e_{s}^{\prime}, \cdot\right\rangle f_{s}^{\prime} d s$, where

$$
e_{s}^{\prime}=\left\{\begin{array}{ll}
e_{s} & \text { if } s \leq t, \\
0 & \text { if } s>t,
\end{array} \quad f_{s}^{\prime}= \begin{cases}f_{s} & \text { if } s \leq t \\
0 & \text { if } s>t\end{cases}\right.
$$

Now, we will define a bounded linear operator $\int_{\mathbb{R}} D_{f_{t}}^{*} \Gamma\left(P_{t}\right) D_{e_{t}} d t$ from $(\mathscr{S}(\mathbb{R}))$ into $(\mathscr{Y}(\mathbb{R}))^{*}$. Before doing this, we find an estimation for the integral $\int_{\mathbb{R}}\left\|D_{f_{t}} \psi\right\|_{k}^{2} d t$, where $k$ is a real number and $\psi \in(\mathscr{S}(\mathbb{R}))$. Let $\psi=\sum_{n=0}^{\infty}\left\langle: \cdot{ }^{\otimes n}:, h_{n}\right\rangle$, where $h_{n} \in E_{c}^{\otimes} n$, for all $n \geq 0$. Each $h_{n}$ can be written as

$$
h_{n}=\sum_{i_{1}, i_{2}, \ldots, i_{n}} a_{i_{1} i_{2} \cdots i_{n}} e_{i_{1}}^{\prime} e_{i_{2}}^{\prime} \cdots e_{i_{n}}^{\prime}
$$

where $a_{i_{1} i_{2} \cdots i_{n}} \in \mathbb{R}$ and $e_{1}^{\prime}, e_{2}^{\prime}, \ldots$ is an orthonormal basis of $E=L^{2}(\mathbb{R})$ given by eigenvectors of the operator $A$ corresponding to the eigenvalues $\lambda_{1}, \lambda_{2}, \ldots$ Also, for any permutation $\sigma$ of the set $\{1,2, \ldots, n\}$, we have $a_{i_{\sigma(1)} i_{\sigma(2)} \cdots i_{\sigma(n)}}=a_{i_{1} i_{2} \cdots i_{n}}$.

For all $t \in \mathbb{R}$ and $n \in \mathbb{N}$, we have

$$
\begin{aligned}
\int_{\mathbb{R}}\left|f_{t} \hat{\otimes}_{1} h_{n}\right|_{k}^{2} d t= & \int_{\mathbb{R}}\left|\sum_{i_{1}, i_{2}, \ldots, i_{n}} a_{i_{1} i_{2} \cdots i_{n}}\left\langle f_{t}, e_{i_{1}}^{\prime}\right\rangle e_{i_{2}}^{\prime} \cdots e_{i_{n}}^{\prime}\right|_{k}^{2} d t \\
= & \int_{\mathbb{R}_{i_{1}, i_{2}, \ldots, i_{n}}} \sum_{j_{1}, j_{2}, \ldots, j_{n}} a_{i_{1} i_{2} \cdots i_{n}} \overline{a_{j_{1} j_{2} \cdots j_{n}}}\left\langle f_{t}, e_{i_{1}}^{\prime}\right\rangle\left\langle f_{t}, e_{j_{1}}^{\prime}\right\rangle \\
& \cdot \lambda_{i_{2}}^{k} \cdots \lambda_{i_{n}}^{k} \lambda_{j_{2}}^{k} \cdots \lambda_{j_{n}}^{k}\left\langle e_{i_{2}}^{\prime}, e_{j_{2}}^{\prime}\right\rangle \cdots\left\langle e_{i_{n}}^{\prime}, e_{j_{n}}^{\prime}\right\rangle d t \\
= & \int_{\mathbb{R}_{i_{1}, i_{2}, \ldots, i_{n}}} \sum_{j_{1}} a_{i_{1} i_{2} \cdots i_{n}} \overline{a_{j_{1} i_{2} \cdots i_{n}}}\left\langle f_{t}, e_{i_{1}}^{\prime}\right\rangle\left\langle f_{t}, e_{j_{1}}^{\prime}\right\rangle \lambda_{i_{2}}^{2 k} \cdots \lambda_{i_{n}}^{2 k} d t \\
= & \int_{\mathbb{R}} \sum_{i_{2}, \ldots, i_{n}} \lambda_{i_{2}}^{2 k} \cdots \lambda_{i_{n}}^{2 k} \sum_{i_{1}} \sum_{j_{1}} a_{i_{1} i_{2} \cdots i_{n}} \overline{a_{j_{1} i_{2} \cdots i_{n}}}\left\langle f_{t}, e_{i_{1}}^{\prime}\right\rangle\left\langle f_{t}, e_{j_{1}}^{\prime}\right\rangle d t \\
= & \int_{\mathbb{R}} \sum_{i_{2}, \ldots, i_{n}} \lambda_{i_{2}}^{2 k} \cdots \lambda_{i_{n}}^{2 k}\left|\sum_{i_{1}} a_{i_{1} i_{2} \cdots i_{n}}\left\langle f_{t}, e_{i_{1}}^{\prime}\right\rangle\right|^{2} d t \\
= & \int_{\mathbb{R}} \sum_{i_{2}, \ldots, i_{n}} \lambda_{i_{2}}^{2 k} \cdots \lambda_{i_{n}}^{2 k}\left|\left\langle f_{t}, \sum_{i_{1}} a_{i_{1} i_{2} \cdots i_{n}} e_{i_{1}}^{\prime}\right\rangle\right|^{2} d t \\
= & \sum_{i_{2}, \ldots, i_{n}} \lambda_{i_{2}}^{2 k} \cdots \lambda_{i_{n}}^{2 k} \int_{\mathbb{R}}\left|\left\langle f_{t}, \sum_{i_{1}} a_{i_{1} i_{2} \cdots i_{n}} e_{i_{1}}^{\prime}\right\rangle\right|^{2} d t \\
\leq & \sum_{i_{2}, \ldots, i_{n}} \lambda_{i_{2}}^{2 k} \cdots \lambda_{i_{n}}^{2 k} M\left|\sum_{i_{1}} a_{i_{1} i_{2} \cdots i_{n} e_{i_{1}}^{\prime}}\right|_{u}^{2} \\
&
\end{aligned}
$$




$$
\begin{aligned}
& =M \sum_{i_{2}, \ldots, i_{n}} \lambda_{i_{2}}^{2 k} \cdots \lambda_{i_{n}}^{2 k} \sum_{i_{1}}\left|a_{i_{1} i_{2} \cdots i_{n}}\right|^{2} \lambda_{i_{1}}^{2 u} \\
& \leq M \sum_{i_{1}, i_{2}, \ldots, i_{n}} \lambda_{i_{1}}^{2 \max (k, u)} \lambda_{i_{2}}^{2 \max (k, u)} \cdots \lambda_{i_{n}}^{2 \max (k, u)}\left|a_{i_{1} i_{2} \cdots i_{n}}\right|^{2} \\
& =M\left|h_{n}\right|_{\max (k, u)}^{2} .
\end{aligned}
$$

Let $v=\max (k, u)$. Then

$$
\begin{aligned}
\int_{\mathbb{R}}\left\|D_{f_{t}} \psi\right\|_{k}^{2} d t & =\int_{\mathbb{R}}\left\|\sum_{n=1}^{\infty}\left\langle: \cdot \otimes(n-1):, n f_{t} \hat{\otimes}_{1} h_{n}\right\rangle\right\|_{k}^{2} d t \\
& =\int_{\mathbb{R}} \sum_{n=1}^{\infty}(n-1) ! n^{2}\left|f_{t} \hat{\otimes}_{1} h_{n}\right|_{k}^{2} d t \\
& =\sum_{n=1}^{\infty} n ! n \int_{\mathbb{R}}\left|f_{t} \hat{\otimes}_{1} h_{n}\right|_{k}^{2} d t \\
& \leq \sum_{n=1}^{\infty} n ! n M\left|h_{n}\right|_{v}^{2} \\
& \leq \sum_{n=1}^{\infty} n ! 2^{n} M\left|h_{n}\right|_{v}^{2} \\
& \leq \sum_{n=1}^{\infty} n ! \lambda_{1}^{2 l n} M\left|h_{n}\right|_{v}^{2} \\
& \leq \sum_{n=0}^{\infty} n ! M\left|h_{n}\right|_{v+l}^{2} \\
& =M\|\psi\|_{v+l}^{2},
\end{aligned}
$$

where $l$ is a large number chosen such that $\lambda_{1}^{2 l} \geq 2$.

We consider the bilinear map $A:(\mathscr{S}(\mathbb{R})) \times(\mathscr{S}(\mathbb{R})) \rightarrow \mathbb{C}$ defined by

$$
A(\varphi, \psi)=\int_{\mathbb{R}}\left\langle\left\langle D_{f_{t}}^{*} \Gamma\left(P_{t}\right) D_{e_{t}} \varphi, \psi\right\rangle\right\rangle d t .
$$

It turns out that $A$ is a continuous bilinear map and, since $(\mathscr{Y}(\mathbb{R}))$ is a nuclear space, by the abstract kernel theorem, there exists a unique bounded operator $B:(\mathscr{Y}(\mathbb{R})) \rightarrow$ $(\mathscr{S}(\mathbb{R}))^{*}$ such that, for all $\varphi, \psi \in(\mathscr{Y}(\mathbb{R}))$, we have

$$
A(\varphi, \psi)=\langle\langle B \varphi, \psi\rangle\rangle .
$$

We denote this operator $B$ by $\int_{\mathbb{R}} D_{f_{t}}^{*} \Gamma\left(P_{t}\right) D_{e_{t}} d t$.

THEOREM 3.11. Using the above notations,

$$
\Gamma(P)=\Gamma(0)+\int_{\mathbb{R}} D_{f_{t}}^{*} \Gamma\left(P_{t}\right) D_{e_{t}} d t .
$$


Proof. Since $\Gamma(P)$ and $\Gamma(0)+\int_{\mathbb{R}} D_{f_{t}}^{*} \Gamma\left(P_{t}\right) D_{e_{t}} d t$ are continuous and linear operators, to prove that they are equal, it is enough to check that they produce the same result when they are applied to exponential functions. For any $\eta \in \mathscr{S}(\mathbb{R})_{c}$, we denote $F_{\eta}=$ : $e^{\langle\cdot, \eta\rangle}$ :. We want to prove that $\Gamma(P) F_{\eta}=\Gamma(0) F_{\eta}+\int_{\mathbb{R}} D_{f_{t}}^{*} \Gamma\left(P_{t}\right) D_{e_{t}} d t F_{\eta}$. To do this, we will use the $S$-transform. For all $\xi \in \mathscr{S}(\mathbb{R})_{c}$, we have

$$
\begin{aligned}
& S\left(\Gamma(0) F_{\eta}+\int_{\mathbb{R}} D_{f_{t}}^{*} \Gamma\left(P_{t}\right) D_{e_{t}} d t F_{\eta}\right)(\xi) \\
& =S\left(\Gamma(0) F_{\eta}\right)(\xi)+S\left(\int_{\mathbb{R}} D_{f_{t}}^{*} \Gamma\left(P_{t}\right) D_{e_{t}} d t F_{\eta}\right)(\xi) \\
& =S(1)(\xi)+S\left(\int_{\mathbb{R}} D_{f_{t}}^{*} \Gamma\left(P_{t}\right) D_{e_{t}} d t F_{\eta}\right)(\xi) \\
& =\left\langle\left\langle 1, F_{\xi}\right\rangle\right\rangle+\left\langle\left\langle\int_{\mathbb{R}} D_{f_{t}}^{*} \Gamma\left(P_{t}\right) D_{e_{t}} d t F_{\eta}, F_{\xi}\right\rangle\right\rangle \\
& =1+\int_{\mathbb{R}}\left\langle\left\langle D_{f_{t}}^{*} \Gamma\left(P_{t}\right) D_{e_{t}} F_{\eta}, F_{\xi}\right\rangle\right\rangle d t \\
& =1+\int_{\mathbb{R}}\left\langle\left\langle\Gamma\left(P_{t}\right) D_{e_{t}} F_{\eta}, D_{f_{t}} F_{\xi}\right\rangle\right\rangle d t \\
& =1+\int_{\mathbb{R}}\left\langle\left\langle\Gamma\left(P_{t}\right)\left\langle e_{t}, \eta\right\rangle F_{\eta},\left\langle f_{t}, \xi\right\rangle F_{\xi}\right\rangle\right\rangle d t \\
& =1+\int_{\mathbb{R}}\left\langle e_{t}, \eta\right\rangle\left\langle f_{t}, \xi\right\rangle\left\langle\left\langle\Gamma\left(P_{t}\right) F_{\eta}, F_{\xi}\right\rangle\right\rangle d t \\
& =1+\int_{\mathbb{R}}\left\langle e_{t}, \eta\right\rangle\left\langle f_{t}, \xi\right\rangle\left\langle\left\langle F_{P_{t} \eta}, F_{\xi}\right\rangle\right\rangle d t \\
& =1+\int_{\mathbb{R}}\left\langle e_{t}, \eta\right\rangle\left\langle f_{t}, \xi\right\rangle e^{\left\langle P_{t} \eta, \xi\right\rangle} d t \\
& =1+\int_{\mathbb{R}}\left\langle e_{t}, \eta\right\rangle\left\langle f_{t}, \xi\right\rangle e^{\int_{-\infty}^{t}\left\langle e_{s}, \eta\right\rangle\left\langle f_{s}, \xi\right\rangle d s} d t \\
& =1+\left.e^{\int_{-\infty}^{t}\left\langle e_{s}, \eta\right\rangle\left\langle f_{s}, \xi\right\rangle d s}\right|_{-\infty} ^{+\infty} \\
& =1+e^{\int_{-\infty}^{+\infty}\left\langle e_{s}, \eta\right\rangle\left\langle f_{s}, \xi\right\rangle d s}-1 \\
& =e^{\int_{-\infty}^{+\infty}\left\langle e_{S}, \eta\right\rangle\left\langle f_{s}, \xi\right\rangle d s} \\
& =e^{\langle P \eta, \xi\rangle} \\
& =\left\langle\left\langle F_{P \eta}, F_{\xi}\right\rangle\right\rangle \\
& =\left\langle\left\langle\Gamma(P) F_{\eta}, F_{\xi}\right\rangle\right\rangle \\
& =S\left(\Gamma(P) F_{\eta}\right)(\xi) \text {. }
\end{aligned}
$$

Application 3.12. Consider the following white noise initial value problem:

$$
\begin{gathered}
\frac{d Q}{d t}=D_{f_{t}}^{*} Q D_{e_{t}} \\
Q(0)=E[\cdot],
\end{gathered}
$$


where $Q:[0, \epsilon) \rightarrow L\left((S(\mathbb{R})),(S(\mathbb{R}))^{*}\right)$ and $E[\cdot]$ denotes the operator that associates to each test function its expectation. Here, $L\left((S(\mathbb{R})),(S(\mathbb{R}))^{*}\right)$ denotes the space of bounded linear maps from $(S(\mathbb{R}))$ into $(S(\mathbb{R}))^{*}$.

This initial value problem is similar to the classical birth-and-death differential equation

$$
\begin{gathered}
\frac{d P}{d t}=(\beta(t)-\delta(t)) P, \\
P(0)=P_{0},
\end{gathered}
$$

where $P(t)$ denotes the size of a population at time $t, \beta(t)$ is the birthrate at time $t$, and $\delta(t)$ is the death rate at time $t$. In the white noise problem, the birthrate $\beta(t)$ is replaced by the creation operator $D_{f_{t}}^{*}$, while the death rate $\delta(t)$ is replaced by the annihilation operator $D_{e_{t}}$. Theorem 3.11 tells us that the solution of the above white noise initial value problem, in the weak sense, is $Q(t)=\Gamma\left(P_{t}\right)$, where $P_{t}=\int_{0}^{t} f_{s}^{*} e_{s} d s$.

\section{REFERENCES}

[1] J. M. C. Clark, The representation of functionals of Brownian motion by stochastic integrals, Ann. Math. Statist. 41 (1970), 1282-1295.

[2] A. A. Dorogovtsev, Conditional measures for diffusion processes and anticipating stochastic equations, Theory Stoch. Process. 4(20) (1998), no. 1-2, 17-24.

[3] I. Kubo and S. Takenaka, Calculus on Gaussian white noise. IV, Proc. Japan Acad. Ser. A Math. Sci. 58 (1982), no. 5, 186-189.

[4] H.-H. Kuo, White Noise Distribution Theory, Probability and Stochastics Series, CRC Press, Florida, 1996.

[5] Y.-J. Lee and H.-H. Shih, The Clark formula of generalized Wiener functionals, Quantum Information, IV (Nagoya, 2001) (T. Hida and K. Saitô, eds.), World Scientific Publishing, New Jersey, 2002, pp. 127-145.

[6] N. Obata, White Noise Calculus and Fock Space, Lecture Notes in Mathematics, vol. 1577, Springer-Verlag, Berlin, 1994.

[7] D. Ocone, Malliavin's calculus and stochastic integral representations of functionals of diffusion processes, Stochastics 12 (1984), no. 3-4, 161-185.

[8] B. Simon, The $P(\phi)_{2}$ Euclidean (Quantum) Field Theory, Princeton Series in Physics, Princeton University Press, New Jersey, 1974.

Said Ngobi: Department of Mathematics and Computer Science, Alabama State University, 915

S. Jackson Street, Montgomery, AL 36101-0271, USA

E-mail address: ngobi@math1ab. a1asu .edu

Aurel Stan: Department of Mathematics, University of Rochester, Ray P. Hylan Building, Rochester, NY 14627, USA

E-mail address: astan@math.nwu.edu 


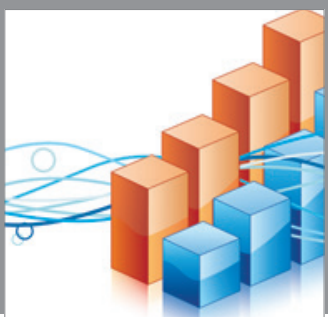

Advances in

Operations Research

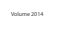

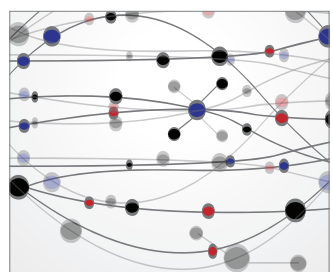

\section{The Scientific} World Journal
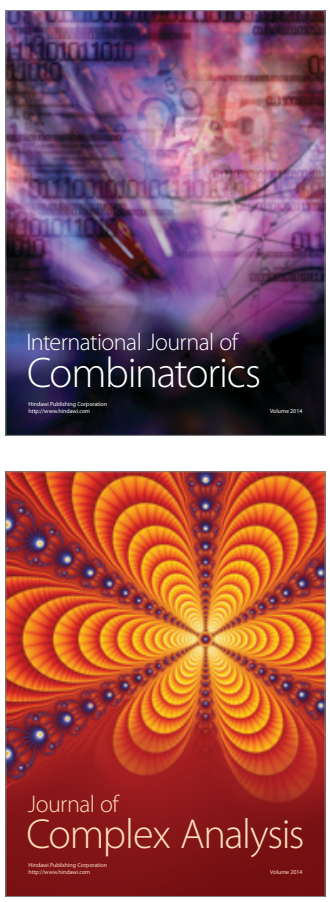

International Journal of

Mathematics and

Mathematical

Sciences
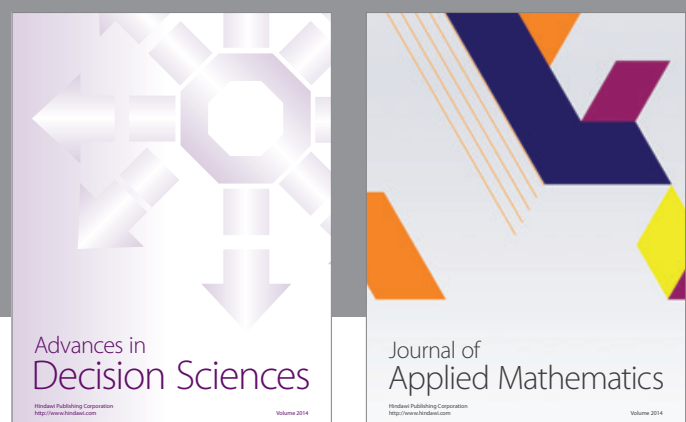

Journal of

Applied Mathematics
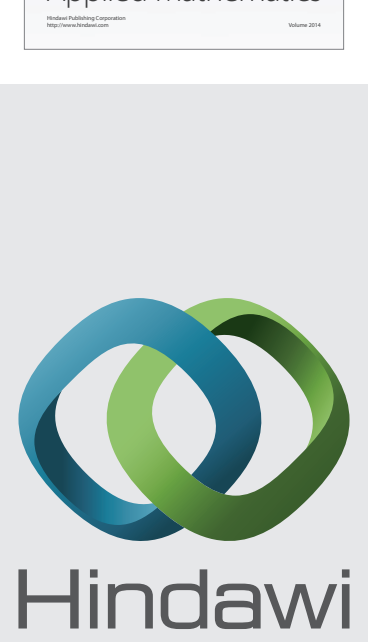

Submit your manuscripts at http://www.hindawi.com
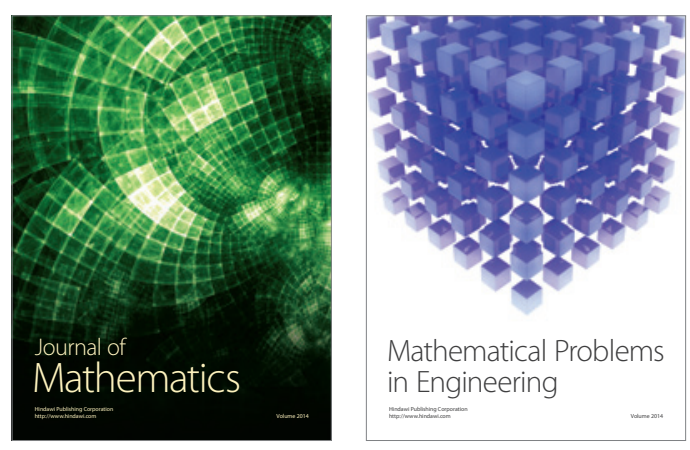

Mathematical Problems in Engineering
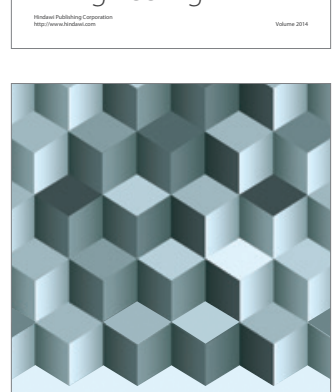

Journal of

Function Spaces
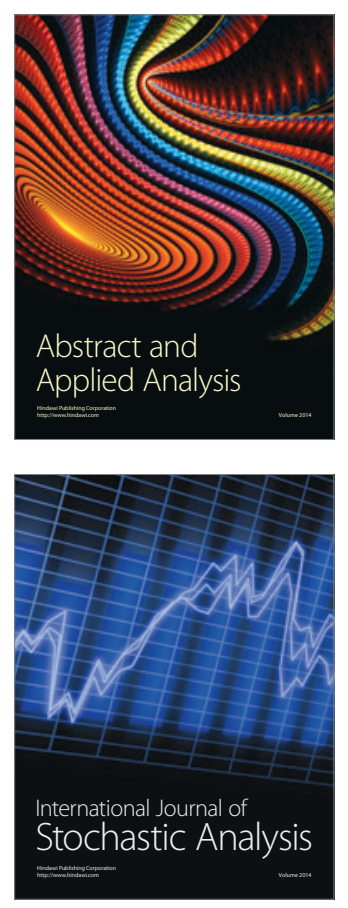

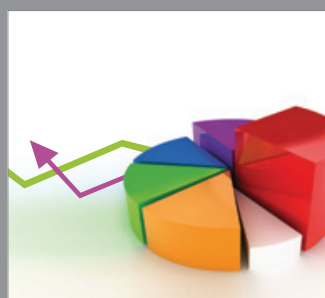

ournal of

Probability and Statistics

Promensencen
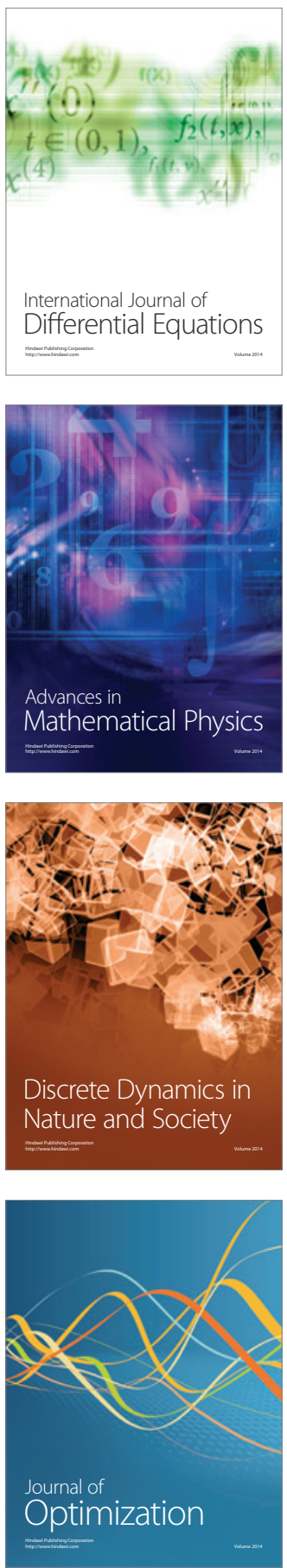